\title{
Characterization of the Graphite Pile as a Source of Thermal Neutrons
}

Zdenek Vykydal ${ }^{1}$, Miloslav Králík ${ }^{1}$, Aleš Jančář ${ }^{2}$, Zdenek Kopecký2 , Jan Dressler², Martin Veškrna²

${ }^{1}$ Czech Metrology Institute, Radiová 1a, CZ-102 00 Praha 10, Czech Republic

2 VF, a.s., Svitavská 588, CZ-679 21 Černá Hora, Czech Republic

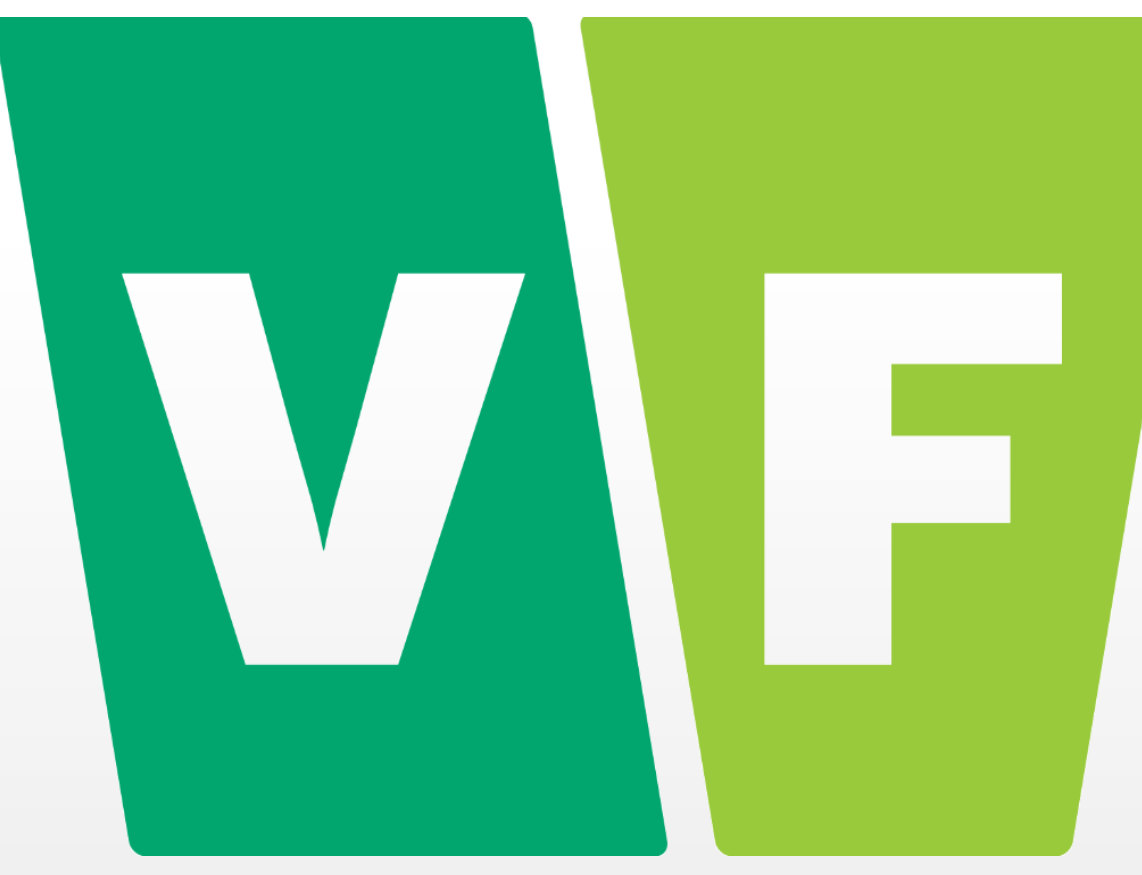

tel: (+420) 266020 299, fax: (+420) 266020 466, e-mail: zvykkydal@cmi.cz, web: www.cmi.cz

\section{Motivation and Methodology}

The absorption cross sections of low energy neutrons obey $1 / v$ law for most materials. Therefore, the detection efficiency of a particular detector increase with decreasing neutron energy. Consequently, most of neutron measuring instruments consist of the detector of thermal neutrons which is located inside of the polyethylene moderator. Development of such instruments requires testing and response callbrations in the field of thermal neutrons. Availability of thermal neutron beams on nuclear reactors is limited and access to them is rather complicated, so it is more convenient to moderate neutrons from the radionuclide neutron sources. Since radiunuclide neutron sources are producing fast neutrons it is necessary to use an appropriate moderator material like heavy water or graphite to thermalize neutrons from the source and to avoid thermal neutron capture in the same time.

Thermal neutron fluence rate is commonly measured by means of gold activation detectors. The quality of a moderated thermal neutron source spectra is then characterized by so called 'cadmium rallo parameter. This parameter is based on properties of the thermal neutron field both bare and encapsulated in a $\mathrm{Cd}$ housing and the correspondig activity ratio characterize the therma

Basic moderated thermal neutron field characteristics are: Cadmium ratio

Cadmium coefficient:

$$
F_{C d}=\frac{R_{C d}-1}{R_{C d}}=\frac{A_{t o t}-A_{e p i}}{A_{t o t}}
$$

Figure 1: Neutron cross-sections of Au-197 and Cd-113

where $A_{\text {tot }}$ is activity of openly irradiated Au foil and $A_{e p i}$ is activity of a Au foil irradiated in the $C d$ capsule. The effective energy cut-off $E_{C d}$ of used cadmium covers is $0.5 \mathrm{eV}$.

\section{Design of the Graphite Pile}

A new graphite pile which should serve as standard source of thermal neutrons has been bulit in the Czech Metrology Institute by the VF, a.s. company. Its actual
dimensions are $1.95 \mathrm{~m}$ (width) $\times 1.95 \mathrm{~m}$ dimensions are $1.95 \mathrm{~m}$ (width) $\times 1.95 \mathrm{~m}$
(lenght) $\times 2.0 \mathrm{~m}$ (height). At the geometrical (lenght) $\times 2.0 \mathrm{~m}$ (height). At the geometrical
centre of the front wall, there is a measurement channel whose dimensions are $0.4 \mathrm{~m} \times 0.4 \mathrm{~m} \times 1.35 \mathrm{~m}$ (depth). The cavity is equipped with a calibration bench, which enables reproducible location of the calibrated device inside of the channel. At the distance of $80 \mathrm{~cm}$ from the cavity axis there are six symmetrically located holes for placement of the radionuclide neutron sources of Am-Be and/or Pu-Be type. The results presented in this work has been taken with three Pu-Be sources placed as depicted in Fig. 2 with the following parameters:

Sources:

प \#1 Pu-Be: $B=8.190 E+7 \mathrm{~s}^{-1} \pm 0.71 \%$ (reference date 13.5.2010)

口 \#2 Pu-Be: $B=4.578 E+7 ~^{-1} \pm 0.70 \%$

(reference date 14.5.2010)

口 \#3 Pu-Be: $B=4.893 E+7 \mathrm{~s}^{-1} \pm 0.71 \%$ (reference date 20.5.2010)

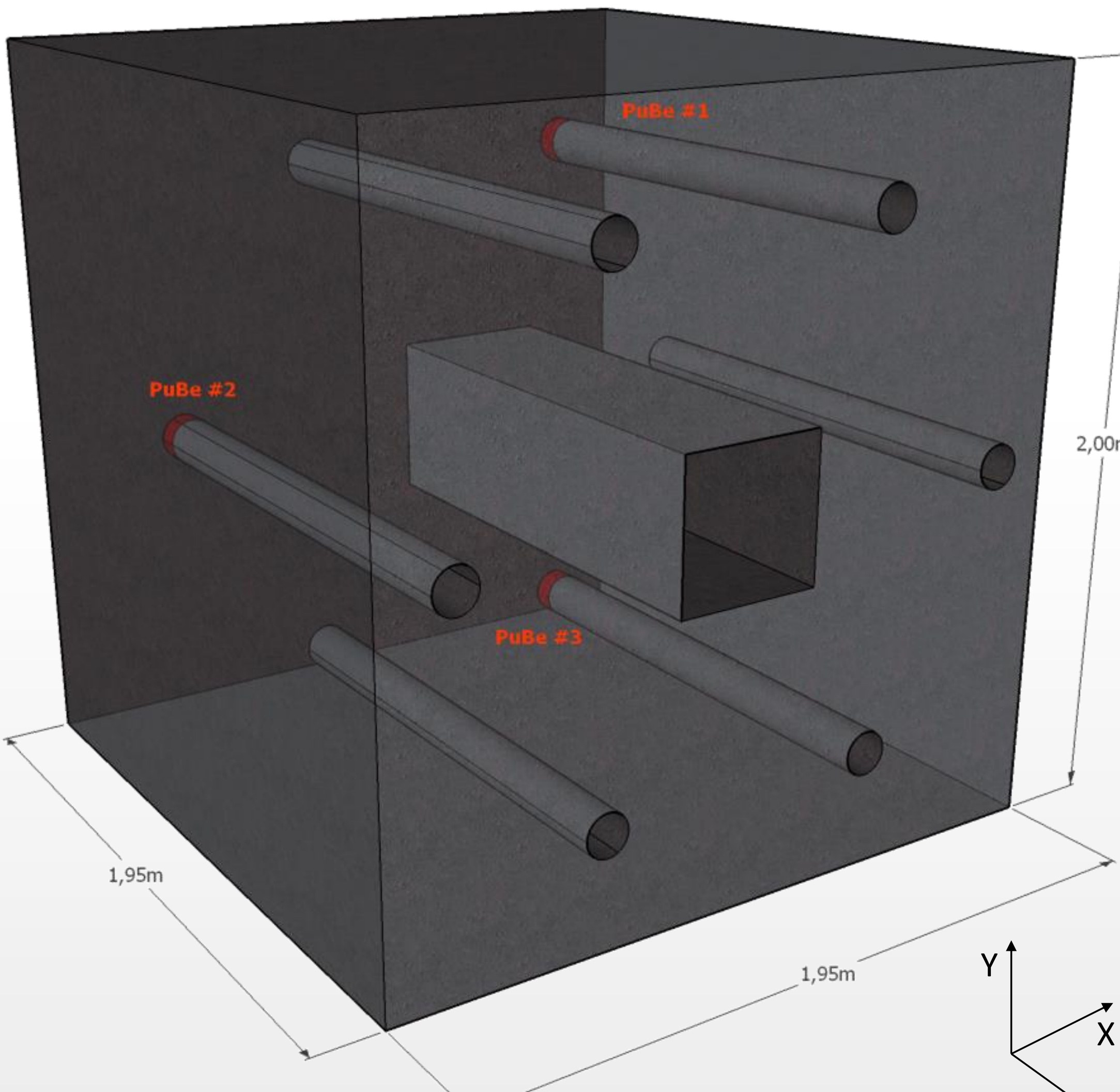

Figure 2: Model of the graphite pile with the position of the sources marked with red color. The coordinate system zero is in the center of the pile.

\section{Absolute Measurement of the Thermal Neutron Fluence Rate}

Absolute values of thermal neutron fluence rate were measured by means of gold foil activation. The $0.1 \mathrm{~mm}$ thick foils with $8 \mathrm{~mm}$ diameter and $99.99 \%$ purity has been used of the pile channel on an array of $3 \times 3$ foils with $10 \mathrm{~cm}$ pitch (see Fig. 3). The mean value of cadmium ratio in the measured (see Fig. 3). The mean value of cadmium ratio in the measured positions is $R_{\mathrm{cd}}$
is $\mathrm{F}_{\mathrm{Cd}}=0.97$.

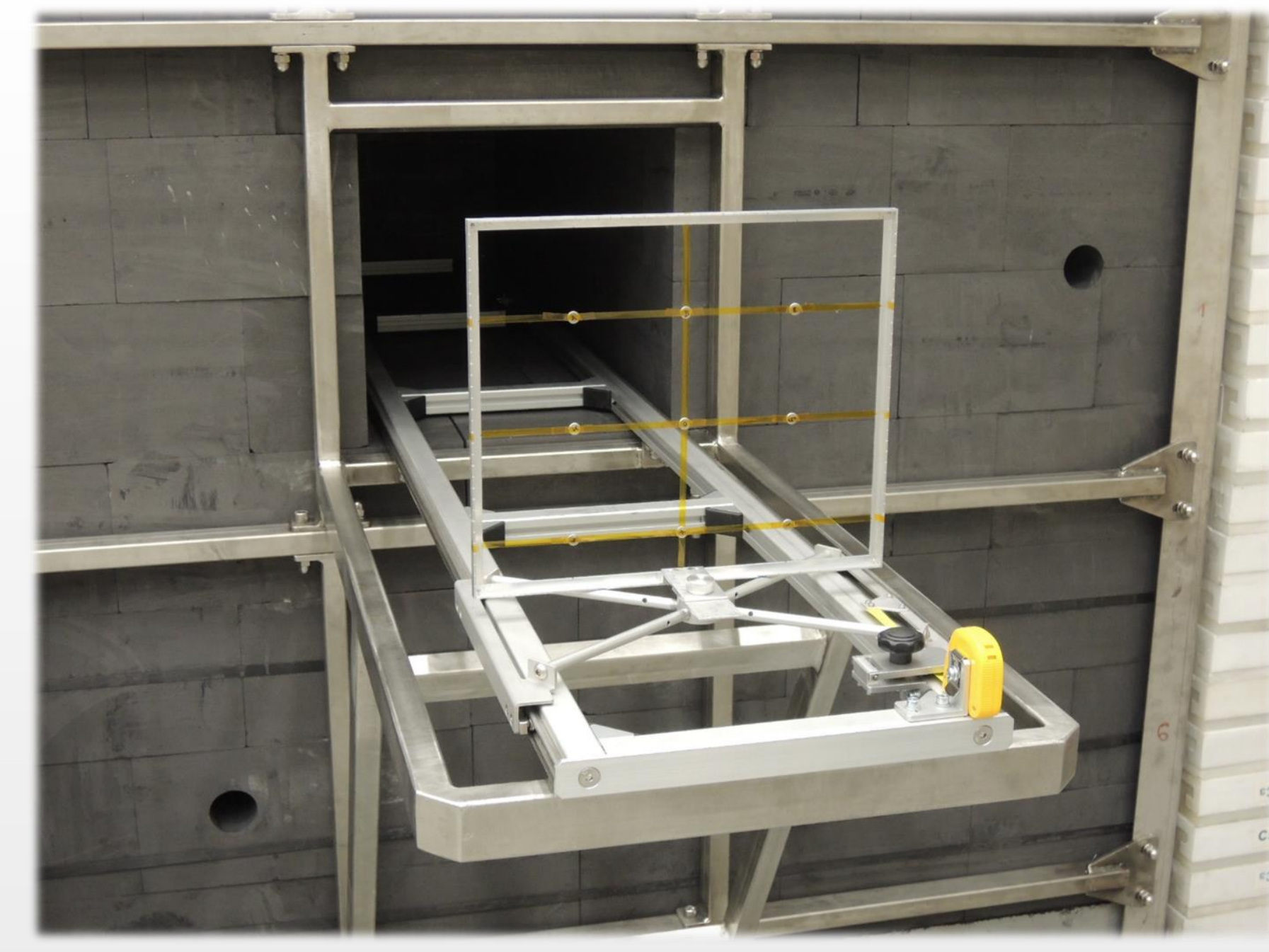

Figure 3: An array of nine Au foils located inside the Cd capsules before placement inside of the thermal neutron

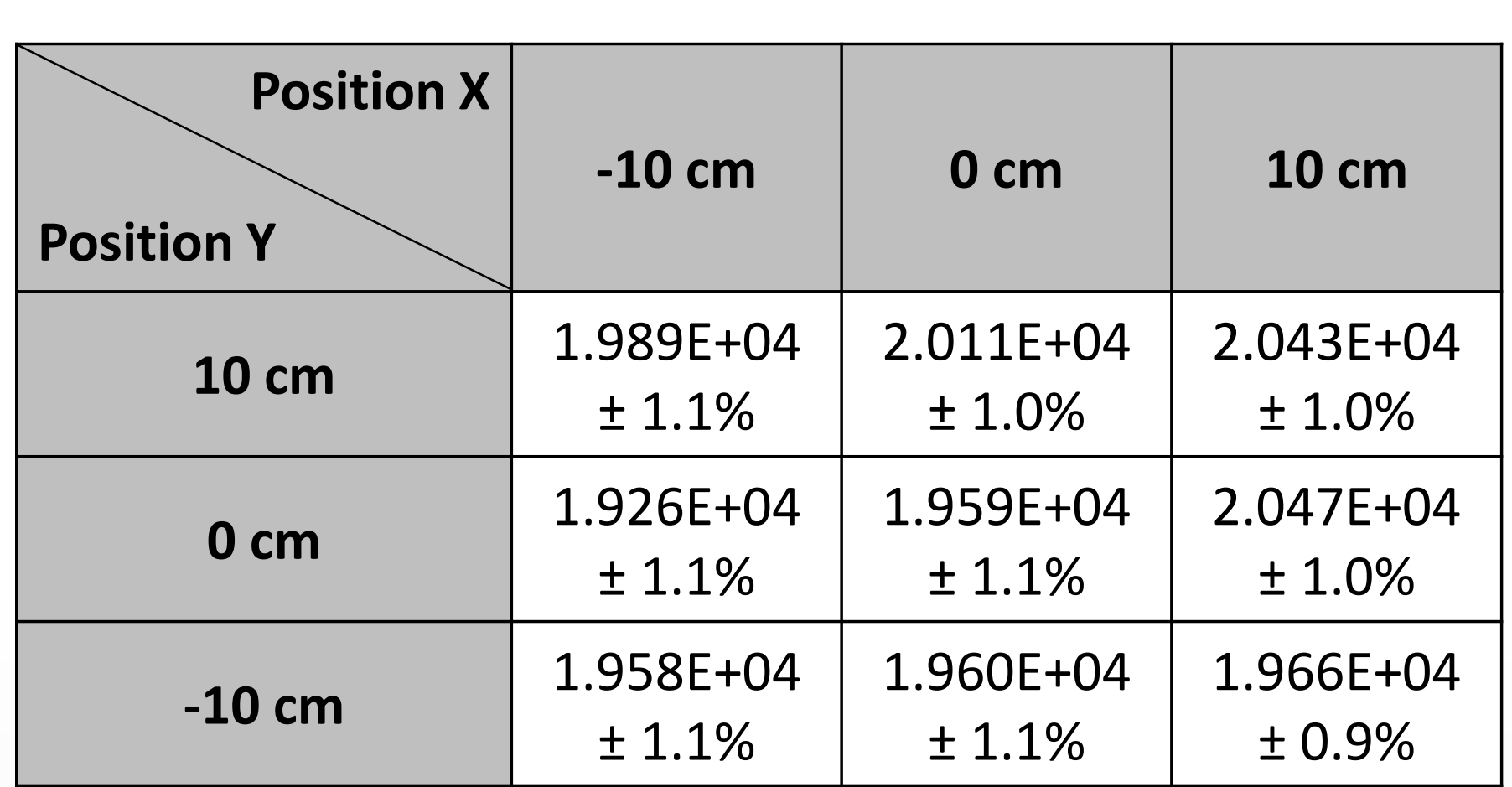
Table 1: Thermal neutron fluence rate distribution at the $x-1$
plane at the position of $-5 \mathrm{~cm}$ from the pile center

\begin{tabular}{|c|c|c|c|}
\hline Position X & $-10 \mathrm{~cm}$ & $\mathbf{0} \mathrm{cm}$ & $10 \mathrm{~cm}$ \\
\hline Position Y & & & \\
\hline \multirow{2}{*}{$10 \mathrm{~cm}$} & $\begin{array}{c}2.004 \mathrm{E}+04 \\
\pm 0.9 \%\end{array}$ & $\begin{array}{c}1.990 \mathrm{E}+04 \\
\pm 1.1 \%\end{array}$ & $\begin{array}{c}2.075 \mathrm{E}+04 \\
\pm 1.0 \%\end{array}$ \\
\hline \multirow{2}{*}{$\mathbf{~ c m}$} & $\begin{array}{c}1.975 \mathrm{E}+04 \\
\pm 1.0 \%\end{array}$ & $\begin{array}{c}1.981 \mathrm{E}+04 \\
\pm 1.0 \%\end{array}$ & $\begin{array}{c}2.004 \mathrm{E}+04 \\
\pm 1.1 \%\end{array}$ \\
\hline \multirow{2}{*}{$-10 \mathrm{~cm}$} & $\begin{array}{c}1.962 \mathrm{E}+04 \\
\pm 1.1 \%\end{array}$ & $\begin{array}{c}1.933 \mathrm{E}+04 \\
\pm 1.1 \%\end{array}$ & $\begin{array}{c}1.988 \mathrm{E}+04 \\
\pm 1.0 \%\end{array}$ \\
\hline
\end{tabular}

Table 2: Thermal neutron fluence rate distribution at the $X-Y$ plane at the position of $5 \mathrm{~cm}$ from the pile center towards the channel opening

\section{Measurement and Simulation of the Field Homogeneity}

The measurement of the thermal neutron fluence rate along the pile cavity axis well demonstrate good agreement of the MCNPX simulation with experimental data. The same simulation has been used to determine the field homogeneity over the
$X-Y$ planes perpendicular to the cavity axis at various $Z$ distances.

Figure 5 show the homogeneity of the thermal neutron field at the central $X-Y$ plane of the pile channel. The thermal neutron fluence rate fluctuate only with $\pm 5 \%$ ar 9 of the plane area. The most deviated points are in the upper left corner of the cavity wher
located.

Evaluation of the field distributions predicted by the simulation at various other positions along the $Z$ axis show that the $5 \%$ homogeneity criteria is fulfilled in the cylindrical volume $40 \mathrm{~cm}$ in diameter around the cavity axis at $\mathrm{Z}$ positions between $\pm 20 \mathrm{~cm}$ from the pile center.

The design of the pile allows to change the position of the sources among 6 available openings. The configuration where the Pu-Be source \#1 will be $\begin{aligned} & \text { located opposite to the sources \#2 and \#3 will be Figure 5: Simulation of the thermal neutron fluence rate homogeneity at the } \\ & \text { investigated in next experiment. }\end{aligned}$
$X-Y$ plane at the central position of the pile channel. The thermal neutron

Map of the Thermal Neutron Fluence Rate Along the Cavity Axis

Measurement of the gold foil activation gives absolute thermal neutron fluence rates but it is detailed measurement of the field homogeneity inside of the pile cavity.

For more detailed measurement we have used two different active detectors:

S Silicon pixel detector Timepix equipped with B-10 converter:

$n+{ }^{10} B \rightarrow \alpha(1.78 \mathrm{MeV})+{ }^{7} L^{*}(1.01 \mathrm{MeV})+\gamma$

$\square$ He-3 gas proportional counter NH05:

$n+{ }^{3} \mathrm{He} \rightarrow p(0.57 \mathrm{MeV})+{ }^{3} \mathrm{H}(0.19 \mathrm{MeV})$

The results of the measurement are presented in Fig. 4 together with the prediction from MCNPX simulation. The results obtained with the active detectors were converted to thermal neutron fluence rates using values measured with activation gold foils.

The results of both experiments and simulation show that thermal neutron fluence rate decrease of about $5 \%$ at distance of $\pm 20 \mathrm{~cm}$ from the pile center.

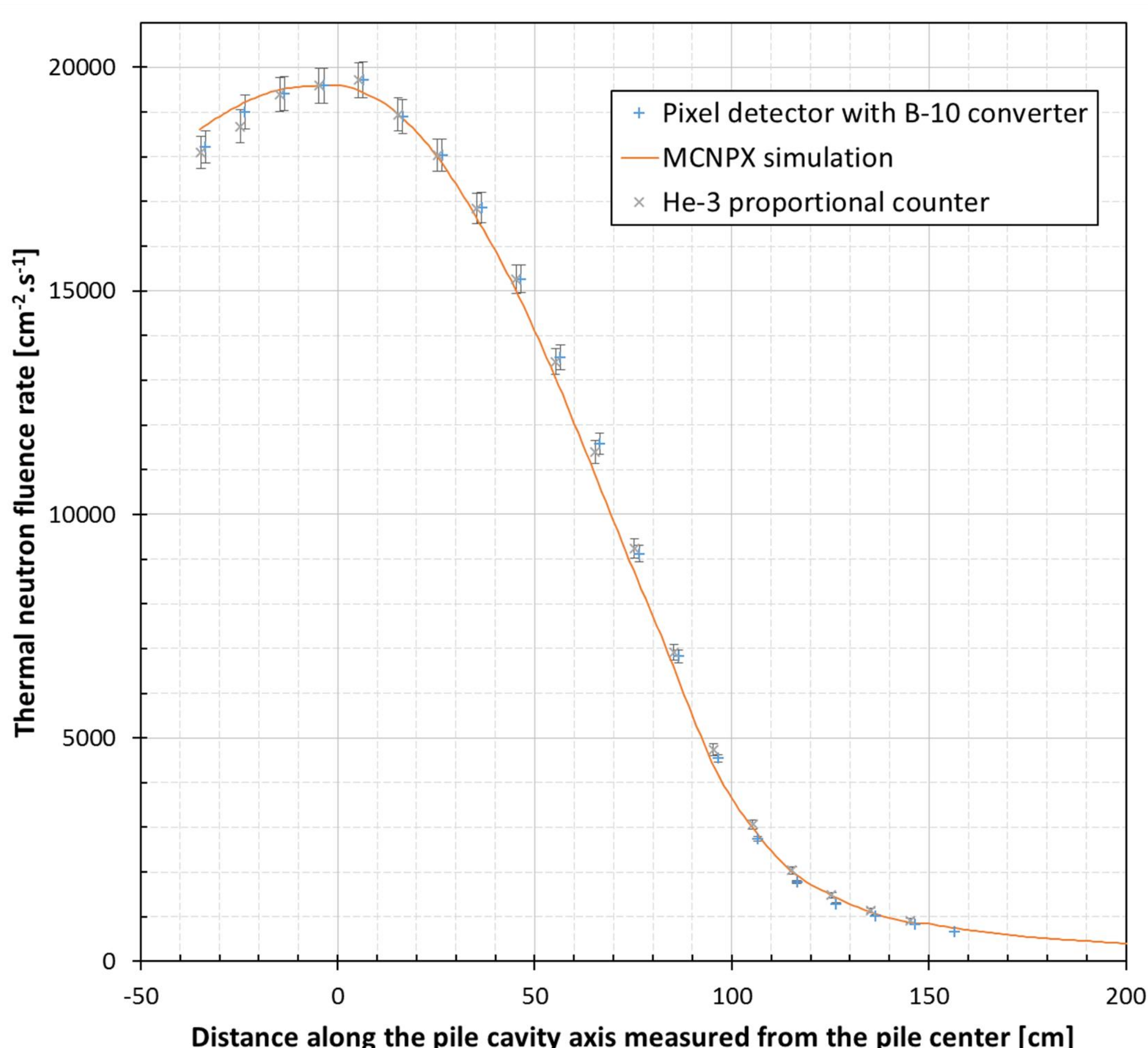

ax. Measurement of the thermal neutron fluence rate along the cavity axis by two different active detectors and it's comparison with the simulation. The pile cavity edge is at the distance of $97.5 \mathrm{~cm}$ from the center.

\section{Summary, Application and References}

General features of the thermal neutron source:

A standard source of thermal neutrons in a form of graphite pile has been built in the Czech Metrology Institute by the VF, a.s. company. The pile has been designed with high flexibility and it can accommodate up to 6 aluminum rods conthing one or more fast neutron sources. Absolute thermal neutron fluence rate and homogeneity has been measured for one particular Pu-Be sources configuration (see Fig. 2) with the following results:

$\square$ Channel dimensions:

Thermal neutron fluence rate: $40 \mathrm{~cm} \times 40 \mathrm{~cm} \times 135 \mathrm{~cm}$ (depth)

Area of $\pm 5 \%$ thermal neutron fl $1.97 \mathrm{E}+04 \mathrm{~cm}^{-2} \cdot \mathrm{s}^{-1}( \pm 1.1 \%)$

Mean cadmium ratio: Cylinder of $40 \mathrm{~cm}$ in diameter and $40 \mathrm{~cm}$ in height centered in the Mean cadmium coefficient:

Applications:

Absolute calibration of the detectors of thermal neutrons is a main application of the pile. The design of the pile allows to change the position of the sources and thus vary with the thermal neutron field parameters within reasonable boundaries, i.e. to maximize field homogeneity or to generate defined field gradient for calibration of large volume neutron detectors.

Calibration of Medipix and Timepix multilayer detectors for measurement of the radiation field spectral composition at the ATLAS experiment at CERN.

Calibration of silicon single-pad detectors with B-10 doping for European Space Agency (ESA).

Calibration of Timepix detectors with Li-6 converter for National Aeronautics and Space Administration (NASA) - used on board

of International Space Station (ISS). 